\title{
Dominant damping effects in friction brake noise, vibration and harshness: the relevance of joints
}

Proc IMechE Part D:

$J$ Automobile Engineering

20I5, Vol. 229(6) 728-734

(C) IMechE 2014

Reprints and permissions:

sagepub.co.uk/journalsPermissions.nav DOI: $10.1177 / 0954407014536378$

pid.sagepub.com

(SAGE

\author{
Merten Tiedemann', Sebastian Kruse ${ }^{2}$ and Norbert Hoffmann ${ }^{1,3}$
}

\begin{abstract}
The experimental analysis of a single component of a brake system and an assembly consisting of three components is used to clarify the relevance of joints in terms of damping and non-linearity in state-of-the-art brake systems. For this purpose a series of experimental modal analyses are conducted. A comparison of the results obtained from the single component and from the assembly strongly indicate that the joints which necessarily exist in an assembled structure have a strong impact on the dynamic behaviour of the structure. The modal damping values of the jointed structure are a factor of up to 60 higher than those of the single-component values. Also, a significant amplitude dependence of the frequency response functions results. The observations demonstrate that joints are a major source of energy dissipation in friction brake systems and, in addition, that they introduce non-linear behaviour to the system which has the potential to limit squeal amplitudes. Therefore, mechanical joints in brake systems should be considered as decisive design elements for noise, vibration and harshness issues in brakes.
\end{abstract}

\author{
Keywords \\ Damping, brake squeal, modelling, joints, noise, vibration and harshness
}

Date received: II November 2013; accepted: 28 April 2014

\section{Introduction}

Friction-induced vibrations in automotive disc brakes are of substantial interest for academic research as well as for industry. ${ }^{1}$ The numerous customer complaints due to brake noise cause high warranty costs in the automotive industry. ${ }^{2}$ To enable silent brakes to be developed, noise, vibration and harshness (NVH) engineers analyse these phenomena using computational and experimental simulations as well as vehicle tests. In the automotive industry, computational simulations have become increasingly important because of shorter product development processes as well as cost reduction necessities. This is also true for the brake system, for which complex eigenvalue analysis has now been established as the standard simulation approach in the industry. ${ }^{3}$ However, numerical simulation results are far from being as reliable as results from brake dynamometers and vehicle tests. Among the variety of unsolved problems concerning the simulations of automotive disc brake squeal, the role of damping and its modelling are crucial. ${ }^{4}$ Until now, damping either has not been modelled or has been inadequately modelled (e.g. Rayleigh damping), even though damping effects mainly determine the stability of the brake system. This is one of the main reasons leading to the well-known problem that computational simulations of the brake system identify more instabilities than can be found in experimental simulations on a brake dynamometer. ${ }^{5}$

Like each complex mechanical structure, an automotive disc brake contains a plethora of joints. Figure 1 highlights a selection of these. In comparison with the effects of material damping, energy dissipation due to mechanical joints in automotive disc brakes seems to have been overlooked for a long time although, in most assembled structures, joint damping is the dominant damping effect. ${ }^{6}$ Moreover, joints introduce a nonlinear behaviour to the brake system, which causes an amplitude dependence and can limit squeal amplitudes.

\footnotetext{
'Hamburg University of Technology, Hamburg, Germany

${ }^{2}$ Audi AG, Ingolstadt, Germany

${ }^{3}$ Imperial College London, London, UK
}

\section{Corresponding author:}

Merten Tiedemann, Hamburg University of Technology, Schlossmuehlendamm 30, Hamburg 21073, Germany.

Email: merten.tiedemann@tuhh.de 


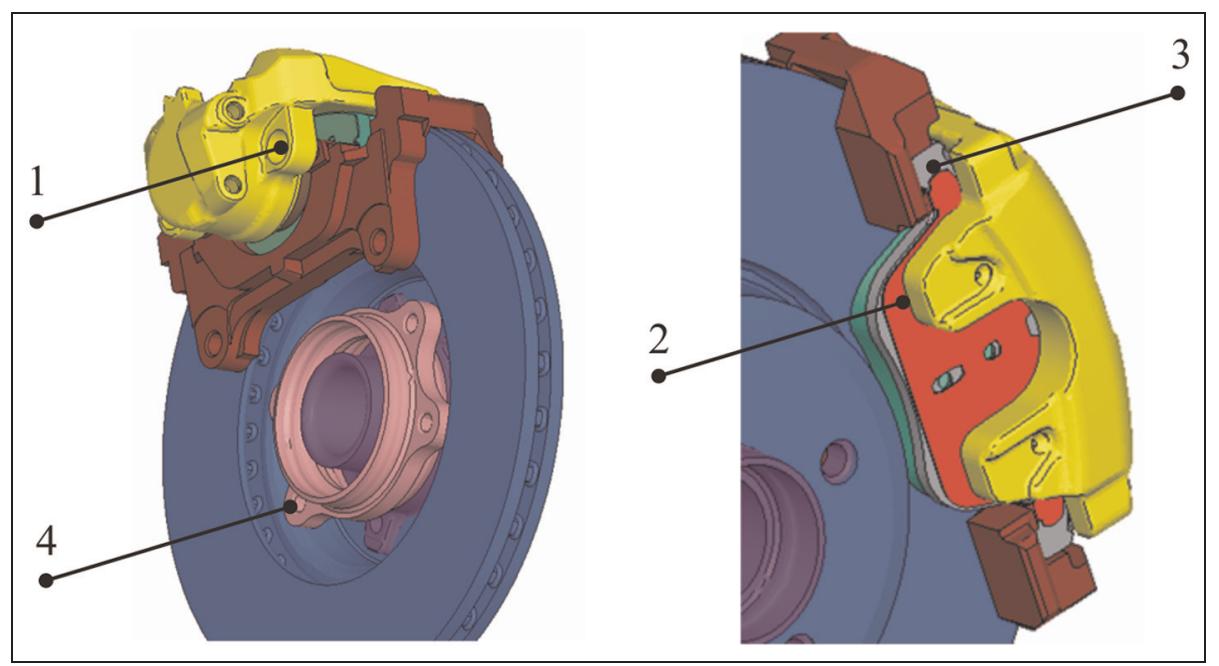

Figure I. Joints in an automotive disc brake: I, calliper-carrier; 2, calliper-pad; 3, pad-carrier; 4, carrier- knuckle.

In other engineering disciplines such as defence or aviation the use of joints as design variables is already at the state-of-the-art level, ${ }^{7,8}$ and joints are effectively used to optimise the dynamic behaviour of the respective mechanical structures. A survey on the use of dry friction for system enhancement has been given, for example, by Ferri. ${ }^{9}$ However, the analysis of the dynamics of joints, from an experimental as well as from a simulation point of view, is known to be challenging. ${ }^{8}$ Ibrahim ${ }^{10}$ has given an overview about the mechanics of contact as well as friction and highlights the main characteristics. Recent investigations ${ }^{11-14}$ have indicated a strong impact of joint damping on the dynamic behaviour of disc brakes. However, further investigations are necessary to determine qualitatively and quantitatively the contribution of joint damping to global damping.

A first step towards the integration of adequate damping models in simulations is the identification of dominant damping effects in the brake system. The objective of this paper is to show the close interrelation of the modelling of damping and the characteristics of mechanical joints as well as to demonstrate that joint damping is the dominant damping mechanism in brake systems. The present work is deliberately focused on the experimental aspects, since there seems to be an imminent need for more and better data on the issues under discussion here. For other present fields of work in the field, which are more conceptual, theoretical or numerical, the recent literature should be consulted (see, for example, the paper by Kruse and Hoffmann ${ }^{15}$ ).

The paper is structured in the following way. In the next section the experimental set-ups and the conducted experiments are introduced. This includes a detailed description of the analysed assembly. The obtained results are analysed and discussed in the third section. The final section provides conclusions and an outlook to future research activities.

\section{Experimental set-up}

To identify the dominant damping effects in automotive disc brakes, two sets of experiments were defined: an experimental modal analysis of the single components of the brake system under free boundary conditions, and an experimental modal analysis of a component assembly. The latter set-up is designed in such a way that additional damping effects imposed by joints in squeal-relevant conditions can be investigated. By comparing the results of both experiments the magnitude of joint damping can be derived.

Among the components in the brake system the brake carrier is a crucial component. It has several contact areas with other components of the brake system and regularly plays a role in NVH countermeasures. In addition, it is close to the contact interface between the brake pad and the disc, which is the origin of brake squeal. Because of these characteristics the brake carrier is used as the guiding component in this paper and is taken as a representative single component of the brake system. However, the statements made in the following about the modal damping and the modal density can be transferred to most of the other components in the brake system. In the following two subsections the set-ups and the conducted experiments are described in detail.

\section{Brake carrier}

An experimental modal analysis of a brake carrier under free boundary conditions was conducted using two triaxial acceleration sensors (PCB 256A01) and a modal hammer (PCB 084A14). The structure was excited at nine points and, for each excitation, six acceleration signals were measured.

\section{Assembly}

For the experimental analysis of joint damping, a tradeoff is required; the set-up should be as simple as 


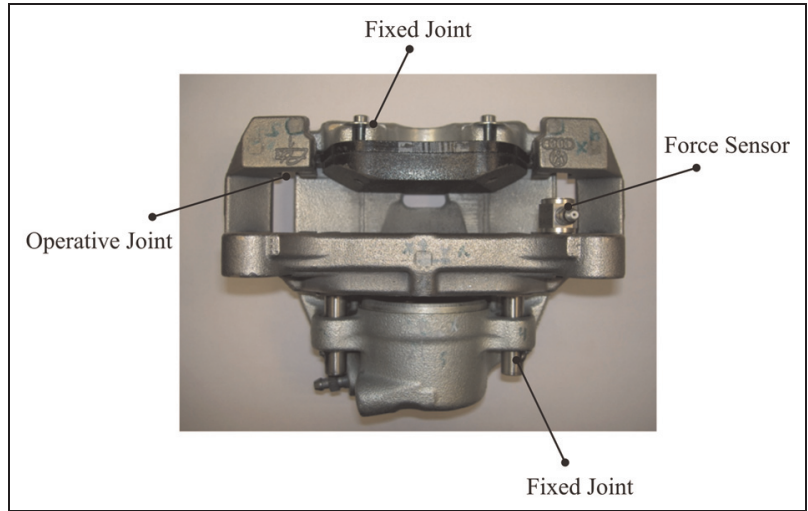

Figure 2. Assembly consisting of the brake carrier, the brake calliper and the brake pad.

possible to enable the assignment of observed damping effects to a specific joint, but it should also be as close as possible to a real brake system. Figure 2 shows the selected minimal assembly used for additional investigations. It consists of a floating calliper, a brake carrier and an outboard brake pad. The assembled structure has three mechanical joints: the contact surface between the brake pad and the carrier arm, the contact surface between the fingers of the brake calliper and the brake pad, and the guidance of the brake calliper in the brake carrier. In order to be able to observe the effect of one specific joint, the other two are deactivated. The rubber bushing normally used between the brake calliper and the brake carrier is removed. Instead a steel bushing is used. To prevent relative movement in the joint the components are connected by a weld spot. The deactivation of the joint between the fingers of the brake calliper and the brake pad is achieved by using bolts (as depicted in Figure 2). The two bolts fix the position of the brake pad relative to the brake calliper. Despite these modifications the general conditions are still comparable with a real brake system concerning the position of the components and the force transmission between them.

The only remaining free joint is the contact surface between the brake pad and the brake carrier (Figure 3). This joint is of special interest since it is often used for $\mathrm{NVH}$ countermeasures (e.g. shim and grease). It should be mentioned that owing to the experimental set-up, which assures a preload-free lining material and low vibration amplitudes of the lining material, the energy dissipated in the lining material does not have a significant impact on the damping level of the assembly. For an experimental modal analysis of a structure with non-linearities (e.g. joints) the choice of the excitation signal is crucial and determines whether the nonlinearity can be studied or not. In this context, the excitation with non-harmonic signals causes a variety of difficulties (e.g. linearisation and uniform excitation of all frequencies) and is not suitable. To identify the effect of the non-linearities the structure is excited with a force-controlled stepped sine signal with a shaker

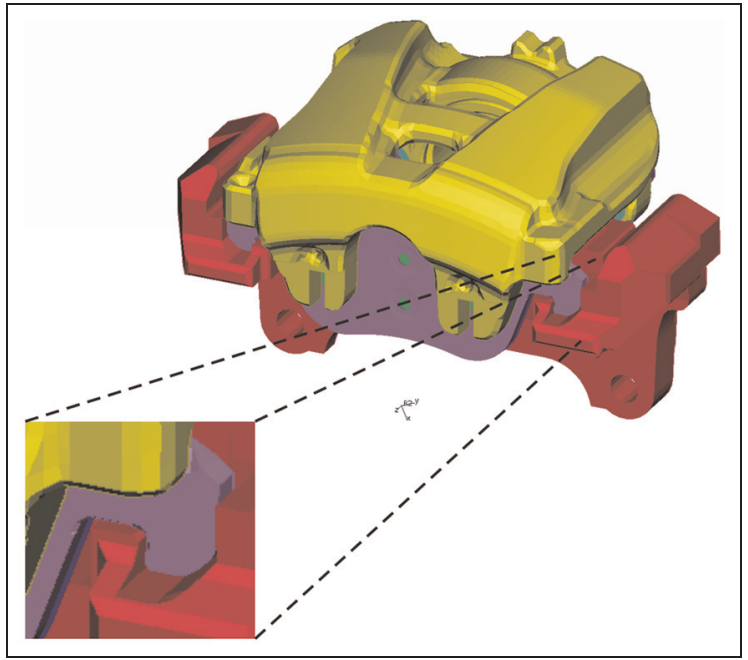

Figure 3. Operative joint (with dry friction).

(type V406). This approach corresponds to the harmonic balance method and allows analysis of non-linear structures; the excitation energy is bundled at one frequency and the harmonic response of the structure is measured in the steady state. ${ }^{16,17}$ The response of the structure is measured via five triaxial acceleration sensors (PCB 256A01) placed on the brake carrier. The position of the force sensor (PCB 208C02) can be seen in Figure 2. The shaker is coupled to the force sensor via a stinger. To minimise the effect of the stinger and the shaker on the measured frequency response functions, several pre-tests with different stinger lengths were conducted. Since the ratio of the effective shaker mass to the mass of the assembled structure is less than $5 \%$, the effect of the shaker mass on the frequency response of the structure is negligible.

\section{Analysis and results}

For analysis of the results the complex mode indicator functions (CMIFs) of the measured data were used since they provide a compact representation of the characteristics of the measurement. The peaks in this function indicate the eigenfrequencies of the system. ${ }^{18}$ The CMIFs of a set of frequency response functions can be obtained by a repeated singular value decomposition of the form

$$
H(\omega)=U(\omega) \Sigma(\omega) V(\omega)
$$

where $H$ is the matrix of frequency responses, $S$ is the matrix containing the singular values and $U$ and $V$ are the left singular eigenvector and the right singular eigenvector respectively. The indicator functions can then be calculated from

$$
\operatorname{CMIF}(\omega)=\Sigma(\omega)^{\mathrm{T}} \Sigma(\omega)
$$

These functions and the modal parameters for the two set-ups are presented in the following two subsections. 


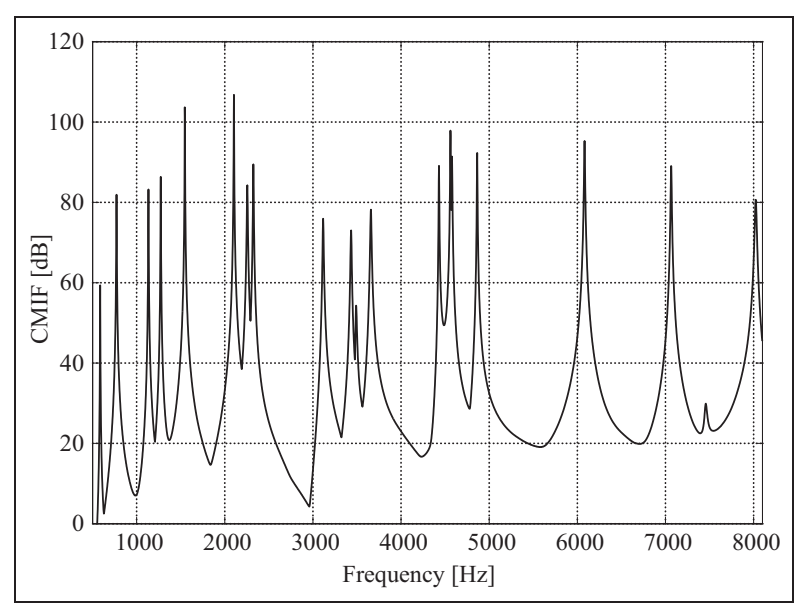

Figure 4. CMIFs of the brake carrier. CMIF: complex mode indicator function.

Table I. Modal parameters of the brake carrier up to $2.4 \mathrm{kHz}$.

\begin{tabular}{lll}
\hline Mode & Eigenfrequency $(\mathrm{Hz})$ & Modal damping $(\%)$ \\
\hline 1 & 586 & 0.21 \\
2 & 772 & 0.25 \\
3 & 1134 & 0.18 \\
4 & 1273 & 0.10 \\
5 & 1547 & 0.08 \\
6 & 2103 & 0.07 \\
7 & 2255 & 0.21 \\
8 & 2324 & 0.15 \\
\hline
\end{tabular}

\section{Brake carrier}

Figure 4 shows the first CMIF of the brake carrier. Up to $2.4 \mathrm{kHz}$, eight eigenfrequencies can be clearly identified, indicating a high modal density. The narrow high peaks emphasise that the modal damping for each of these modes is low.

Table 1 shows the modal parameters extracted from the measured frequency response functions up to $2.4 \mathrm{kHz}$. The above-emphasised idea of low modal damping is correct; the obtained values prove that the brake carrier is lightly damped. This is not surprising since, in the case of a single component, modal damping represents mainly the material damping of a structure, which is small for all metals.

\section{Assembly}

Figure 5 shows the CMIF of the assembly for the frequency range between $1000 \mathrm{~Hz}$ and $1500 \mathrm{~Hz}$ for an excitation level of $0.5 \mathrm{~N}$. The shape of the peaks indicates that the modal damping for each of the modes in this frequency range is significant.

Table 2 shows the modal parameters of the assembled structure up to $2.4 \mathrm{kHz}$ extracted from the measured frequency response functions for an excitation level of $0.5 \mathrm{~N}$. This low-frequency range is of special interest since with increasing frequency the

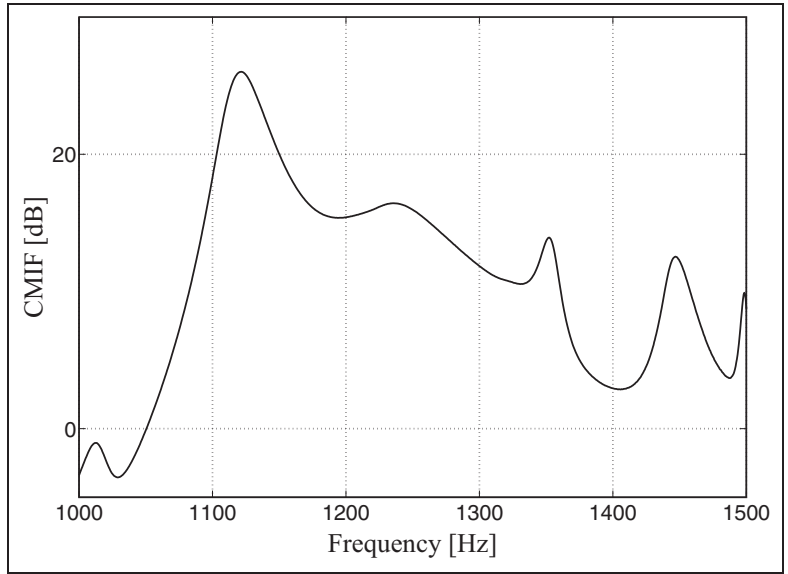

Figure 5 CMIFs of the brake carrier. CMIF: complex mode indicator function.

Table 2. Modal parameters of assembly for an excitation level of $0.5 \mathrm{~N}$ up to $2.4 \mathrm{kHz}$.

\begin{tabular}{lll}
\hline Mode & Resonance frequency $(\mathrm{Hz})$ & Modal damping $(\%)$ \\
\hline 1 & 597 & 0.66 \\
2 & 708 & 0.88 \\
3 & 1013 & 1.27 \\
4 & 1117 & 1.8 \\
5 & 1235 & 6.02 \\
6 & 1354 & 0.65 \\
7 & 1444 & 0.83 \\
8 & 1583 & 2.43 \\
9 & 1846 & 1.77 \\
10 & 2019 & 1.09 \\
11 & 2128 & 0.39 \\
12 & 2184 & 0.67 \\
13 & 2227 & 0.9 \\
14 & 2264 & 1.08 \\
15 & 2315 & 1.03 \\
\hline
\end{tabular}

displacement amplitudes decrease, leading to a reduced impact of the joints on the global damping.

The number of resonance frequencies in the analysed frequency range changed from eight to 15 in comparison with the brake carrier as a single component. The origin of these additional modes is twofold. First, new mode shapes occur owing to the changed boundary conditions. Second, it is a well-known fact that the deflection shapes of a brake system in the squealing state are often dominated by single-component mode shapes which have been observed under free boundary conditions. ${ }^{1}$ In this case, the brake calliper and the brake pad impose a single-component mode shape on the structure mode shape. Keeping this in mind, at least the mode shapes of the assembled structure at $597 \mathrm{~Hz}$, $708 \mathrm{~Hz}, 1117 \mathrm{~Hz}, 1235 \mathrm{~Hz}$ and $1583 \mathrm{~Hz}$ can be assumed to be similar to the mode shapes of the brake carrier found under free boundary conditions (see Table 1).

Not only do the resonance frequencies and their quantity change but also so does the modal damping. 


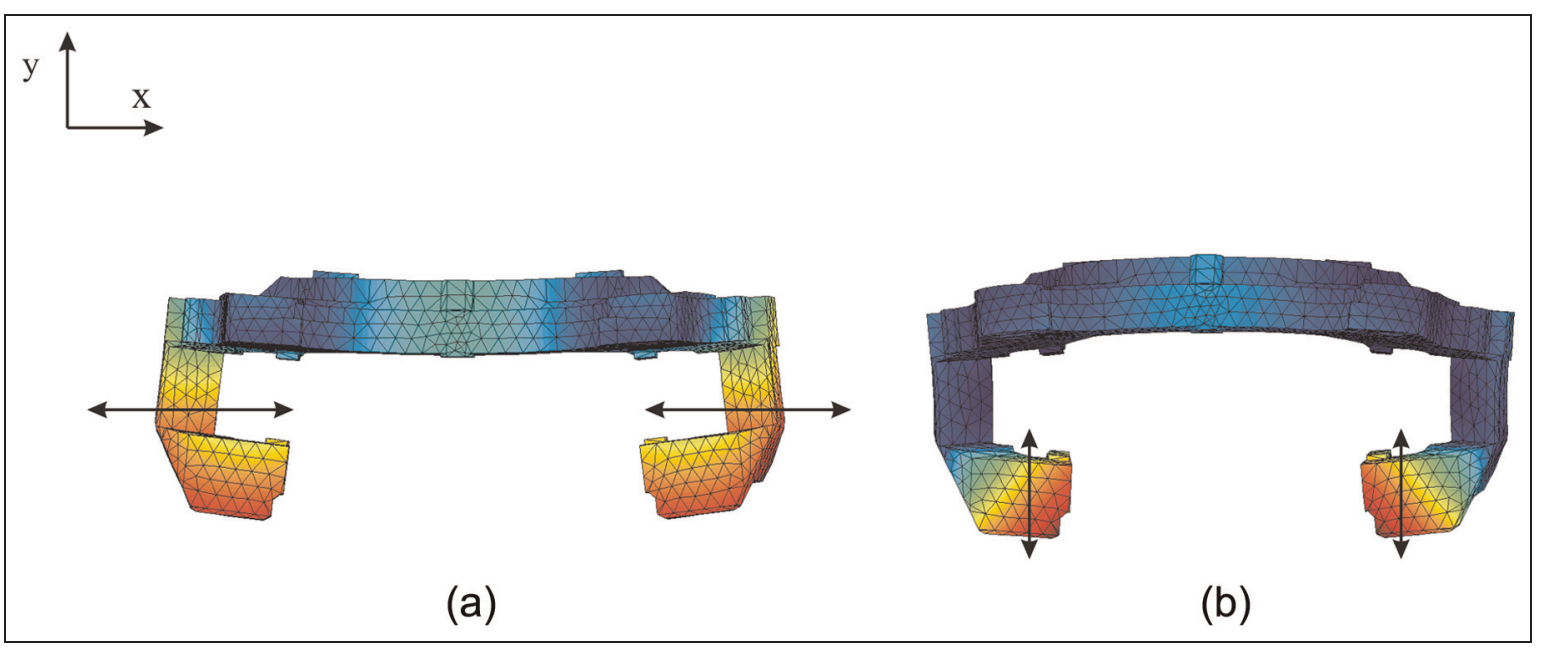

Figure 6. (a) First mode shape and (b) fifth mode shape (right) of the brake carrier (top view).

When the modal damping values listed in Table 1 and Table 2 are compared, it can be observed that the modal damping increased significantly. Taking the modes of the assembled structure which were identified as dominated by the brake carrier mode shapes and comparing their modal damping values with the respective values of the brake carrier delivered additional insights into the characteristics of joint damping; the increment in damping is not uniform. For the different modes it varies between a factor of 3 and a factor of 60. The influence of joints on global damping is mode dependent.

To explain this observation, the mode shapes of the brake carrier under free boundary conditions are addressed again. Figure 6 shows the first mode shape and the fifth mode shape of the brake carrier, which relate to the first mode and eighth mode respectively of the assembled structure.

The first mode shape is characterised by a movement of the entire carrier arms in the $x$ direction, whereas the fifth mode shape shows a huge movement of the end parts of the arms in the $y$ direction. The joint between the brake pad and the brake carrier allows the relative displacement in the $y$ direction and limits the relative displacement in the $x$ and $z$ directions. Since dry friction effects in the contact surface cause energy dissipation in the joint, large amplitudes of the relative displacement increase the quantity of dissipated energy.

On looking at Tables 1 and 2 again and comparing the increments in the modal damping of the first mode and in the modal damping of the fifth mode of the brake carrier with their respective counterparts of the assembly, it can be seen that the modal damping of the former mode is increased by a factor of 3 , whereas the modal damping of the latter mode is increased by the factor of about 30 . These results clearly indicate the relation between the displacements in the joint and their impacts on the modal damping.

Although a significant amount energy is dissipated in joints, these non-proportional damping effects can

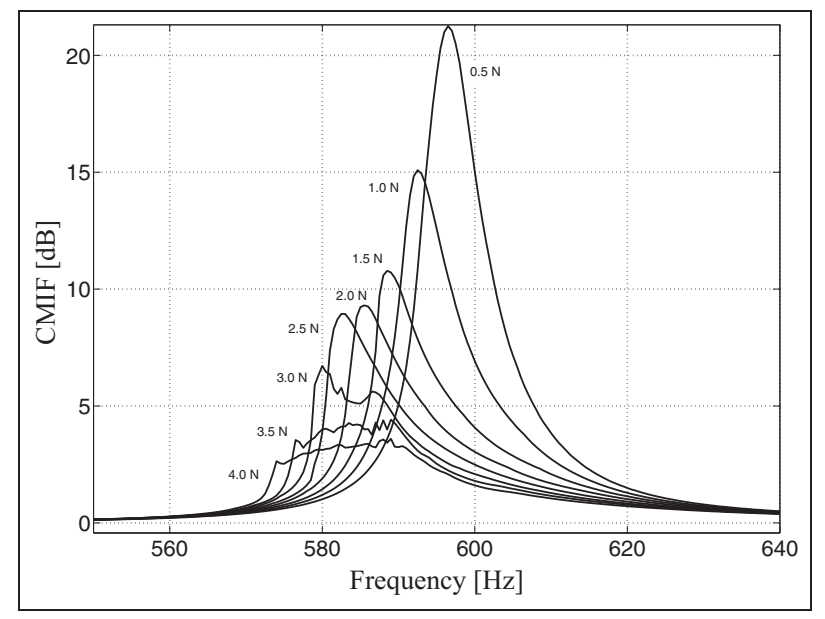

Figure 7. CMIFs for different excitation levels. CMIF: complex mode indicator function.

lead to destabilisation of the equilibrium solution. This phenomenon is well known and has been extensively discussed by Hoffmann and $\mathrm{Gaul}^{4}$ and Fritz et al. ${ }^{19}$ Sinou and Jezequel ${ }^{20}$ have also discussed this phenomenon and additionally considered the limit cycle amplitudes in a friction-excited system. Their results revealed that the influences of non-proportional damping on the stability behaviour of the equilibrium solution and on the limit cycle amplitudes is complex.

Because of the non-linear characteristics of the joint, different excitation amplitudes lead to a different response of the system. In order to analyse this amplitude dependence of the dynamics of the assembled structure, tests with different force amplitudes were conducted. Figure 7 and Figure 8 show the CMIFs for different excitation levels in three different frequency ranges.

The figures clearly demonstrate that the modal parameters, namely the resonance frequency and the modal damping, are both amplitude dependent for all analysed modes. The CMIF of the first mode, depicted in 


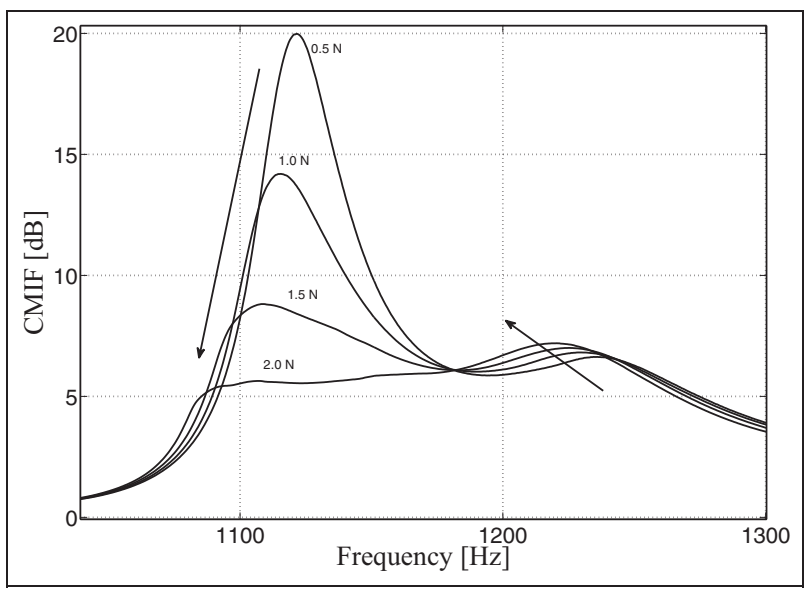

Figure 8. CMIFs for different excitation levels. CMIF: complex mode indicator function.

Figure 7, shows a clear shift in the resonance frequency to lower frequencies. In addition, a decreasing amplitude of the CMIF for higher force amplitudes can be observed. This indicates an increment in the modal damping. Figure 8 shows the CMIF in the frequency range where the fourth and the fifth modes can be found. Whereas the resonance frequency decreases for both with increasing excitation level, the modal damping behaves differently. It increases for the fourth mode and decreases for the fifth mode.

\section{Conclusion and outlook}

It was shown that the modal damping of the assembly consisting of a brake carrier, a brake calliper and a brake pad is significantly higher than the modal damping of the brake carrier. The additional modal damping is introduced by the studied joint. Hence, joint damping is one of the dominant damping effects in automotive disc brakes. As a consequence, it seems as if material damping could rather be neglected in modelling but not in joint damping. In addition, the results indicate that joint damping is highly mode dependent. A nonproportional damping effect such as joint damping can stabilise or destabilise the equilibrium solution. Taking into consideration joint damping in the stability calculations of the equilibrium solutions would definitely increase their validity.

A further result of this study is that joints introduce non-linearities to the brake system. From a stability point of view this fact can be even more valuable than the strong energy dissipation in mechanical joints. In fact, non-linearities determine the bifurcation behaviour and the evolution of non-equilibrium solutions.

The implications for application and future work are twofold. First, modelling of damping should not focus on material parameters alone, but also on adequate modelling of joint damping. This is possible either by using measured or estimated modal damping parameters of the brake system (or parts of it) or by using proper joint models. Second, a systematic use of joints for the reduction in the brake noise problems could be a target. Although the brake community is aware of the potential of joints, until now the corresponding opportunities have not yet been used consistently, and the industry instead strongly concentrates on structural and material modifications alone. Further work will focus on these two aspects in order to improve the quality of disc brake simulations. To achieve this, the joints in disc brakes will be analysed by further experiments, and characteristic parameters will be identified. These can then hopefully be used for adequate modelling of joints and will work as a novel basis for NVH countermeasures in brake squeal.

\section{Declaration of conflict of interest}

The author declares that there is no conflict of interest.

\section{Funding}

Part of this work was supported by Audi AG and AiF in the context of Cooperative Industrial Research (Industrielle Gemeinschaftsforschung) (grant number $16799 \mathrm{~N})$.

\section{References}

1. Hoffmann N and Gaul L. Friction induced vibrations of brakes - research fields and activities. SAE paper 200801-2578, 2008.

2. Akay A. Acoustics of friction. J Acoust Soc Am 2002; 4: 1525-1548.

3. Kinkaid NM, O'Reilly OM and Papadopoulos P. Automotive disc brake squeal. J Sound Vibr 2003; 267: 105-166.

4. Hoffmann $\mathrm{N}$ and Gaul L. Effects of damping on modecoupling instability in friction induced oscillations. $Z$ Angew Math Mech 2003; 83(8): 524-534.

5. Ouyang H, Nack W, Yuan Y and Chen F. Numerical analysis of automotive disc brake squeal: a review. Int $J$ Veh Noise Vibr 2005; 1(3-4): 207-231.

6. Gaul L and Nitsche R. The role of friction in mechanical joints. Appl Mech Rev 2001; 54(2): 93-105.

7. Ewins DJ. The importance of joints on the dynamics of gas turbine structures. In: SNL/NSF International Workshop on Joint Mechanics, Arlington, Virginia, USA, 16-18 October 2006, Report SAND2007-7761, Sandia National Laboratories, Albuquerque, New Mexico, USA, January 2008: 23-32.

8. Segalman DJ, Gregory DL, Starr MJ et al. Handbook on dynamics of jointed structures. Report, Sandia National Laboratories, Albuquerque, New Mexico, USA, June 2009.

9. Ferri AA. Friction damping and isolation systems. Trans ASME, J Vibr Acoust 1995; 117: 196-206.

10. Ibrahim RA. Friction-induced vibration, chatter, squeal, and chaos - Part I. Appl Mech Rev 1994; 47(7): 209-226.

11. Jerschl M, Suess D, Kruse $S$ and Willner K. Investigation of the dynamic behaviour of braking disc joints. In: Eurobrake 2012, Dresden, Germany, 16-18 April 2012. London: FISITA.

12. Kruse S, Reuss $\mathbf{P}$ and Hoffmann N. The influence of nonlinear joint characteristics on friction-induced vibrations. 
In: 83rd annual meeting of the International Association of Applied athematics and Mechanics (Gesellschaft für Angewandte Mathematik und Mechanik), Darmstadt, Germany, 26-30 March 2012.

13. Kruse S, Zeumer B, Reuss P et al. Detection of critical instabilities in brake squeal on nonlinear joint characteristics. In: Eurobrake 2012, Dresden, Germany, 16-18 April 2012. London: FISITA. Paper number: EB2012-MS-29.

14. Kruse S, Tiedemann M, Zeumer B et al. The influence of joints on friction induced vibrations in brake squeal. $J$ Sound Vibr 2014 (submitted).

15. Kruse $\mathrm{S}$ and Hoffmann NP. On the robustness of instabilities in friction-induced vibration. Trans ASME, $J$ Vibr Acoust 2013; 135: 06103.

16. Blohm PW. Untersuchung des nichtlinearen Übertragungsverhaltens von Strukturen mit lokalen Fügestellen
[Investigation of the nonlinear transfer behavior of structures with local joints]. Fortschritt-Berichte VDI-Reihe 11, No. 164, VDI-Verlag, Düsseldorf, Germany, 1992.

17. Eibelshaeuser P. Rechnergestützte experimentelle Modalanalyse mittels gestufter Sinusanregung [Computer-based experimental modal analysis with stepped sine excitation]. Berlin: Springer, 1990.

18. Ewins DJ. Modal testing: theory, practice and application. Baldock, Hertfordshire: Research Studies Press, 2000.

19. Fritz G, Sinou JJ, Duffal J-M and Jézéquel L. Investigation of the relationship between damping and modecoupling patterns in case of brake squeal. J Sound Vibr 2007; 307: 591-609.

20. Sinou JJ and Jezequel L. The influence of damping on the limit cycles for a self-exciting mechanism. J Sound Vibr 2007; 304: 875-893. 